\title{
Congenital dilatation of the common bile duct and pancreaticobiliary maljunction-clinical implications
}

\author{
Wataru Kimura
}

Received: 17 March 2008/Accepted: 18 March 2008/Published online: 19 August 2008

(C) The Author(s) 2008

\begin{abstract}
Clinical conditions and diagnosis Congenital dilatation of the common bile duct is a disease in which the extrahepatic bile duct, or both the extra and intrahepatic bile ducts, is dilated in various ways. Pancreaticobiliary maljunction is a disease in which the pancreatic duct meets the bile duct outside of the duodenal wall beyond the sphincter Oddi. Recently, these diseases have been thought to be closely related to each other but to be different malformations. Biliary tract carcinoma, especially bile duct carcinoma, is found in about $30 \%$ of patients with congenital dilatation of the bile duct. The concomitance of bile and pancreatic juice and their stasis in the biliary tract induce cellular proliferation and reproduction and stimulate genetic alterations in biliary epithelium, which may play an important role in carcinogenesis of the bile duct.

Therapeutic strategies Endoscopic retrograde cholangiopancreatography is useful for examining pancreaticobiliary maljunction. The operation is dilated bile duct resection and hepaticojejunostomy, which ensure that pancreatic juice and bile do not mix in the bile duct. Gallbladder carcinoma develops in more than $90 \%$ of pancreaticobiliary maljunction without bile duct dilatation.
\end{abstract}

Keywords Pancreaticobiliary maljunction .

Congenital dilatation of the common bile duct .

The sphincter Oddi · Bile duct carcinoma .

Carcinogenesis of the bile duct · Gallbladder carcinoma

\section{W. Kimura $(\bowtie)$}

Department of Gastroenterological and General Surgery,

Yamagata University School of Medicine,

2-2-2 Iida-Nishi,

Yamagata, Yamagata 990-9585, Japan

e-mail: wkimura@med.id.yamagata-u.ac.jp

\section{Congenital dilatation of the common bile duct}

Clinical conditions and diagnosis

\section{Clinical conditions}

Congenital dilatation of the common bile duct is a disease in which the extrahepatic bile duct, or both the extra and intrahepatic bile ducts, is dilated in various ways, from a spindle form (Fig. 1) to a cystic form [1] (Figs. 2 and 3). Typically, the common bile duct dilates cystically, which is why this is also called choledochal cysts.

Although this disease is quite rare, it has been noticed in relation to pancreaticobiliary maljunction (Fig. 4), based on advances in diagnostic techniques involving imaging or endoscopy, such as endoscopic retrograde cholangiopancreatography (ERCP) or magnetic resonance cholangiopancreatography (MRCP).

One-third of the reported cases in the world are Japanese. The disease is found five to seven times more often in Asians than in Caucasians. Although the disease is found in children and the elderly, most cases are found before 30 years of age. The male-to-female ratio is 1:3.

With regard to the classification, the Alonso-Lej classification [2] is notable: the shape of the dilated bile duct is divided into three categories, i.e., a cystic type (type I), a diverticulum type (type II), and a cyst of the papilla of Vater (type III). However, types II and III are very rare. Todani's classification [3], which is based on this one, is used worldwide.

Various theories with regard to its pathogenesis have been reported, such as a possible imbalance in epithelial proliferation of the common bile duct, or stenosis of the lower bile duct. In 1968, Babbit [4] drew attention to the 


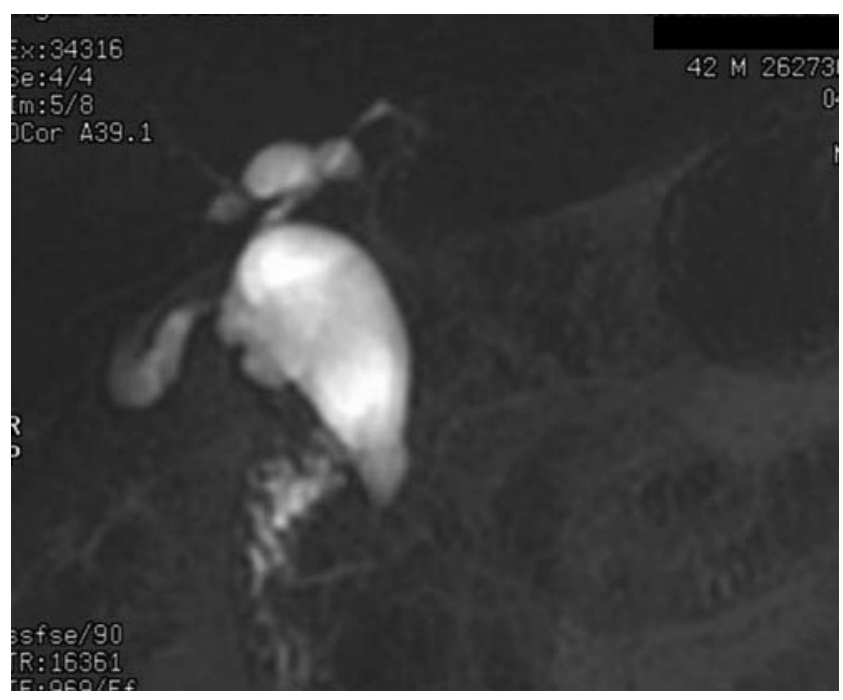

Fig. 1 MRCP of congenital dilatation of the common bile duct of a spindle form

direct relation between congenital dilatation of the bile duct and pancreaticobiliary maljunction.

Pancreaticobiliary maljunction is a disease in which the pancreatic duct meets the bile duct outside of the duodenal wall beyond the sphincter Oddi. Under this condition, the pancreatic juice flows into the bile duct by a pressure gradient [5]. The inflow of pancreatic juice is confirmed by an elevation of amylase values in the bile. Because pancreaticobiliary maljunction is found in almost $100 \%$ of the cases of type I congenital bile duct dilatation, the inflow of pancreatic juice into the bile duct was thought to cause dilatation of the bile duct.

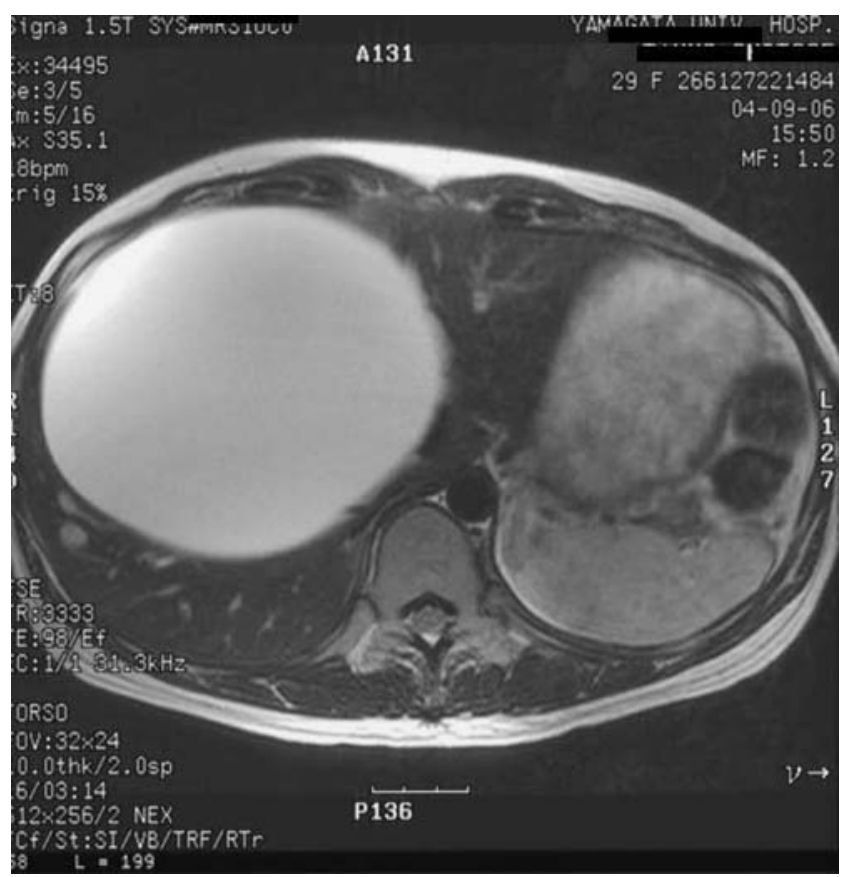

Fig. 2 MRI of congenital dilatation of the common bile duct of a cystic form

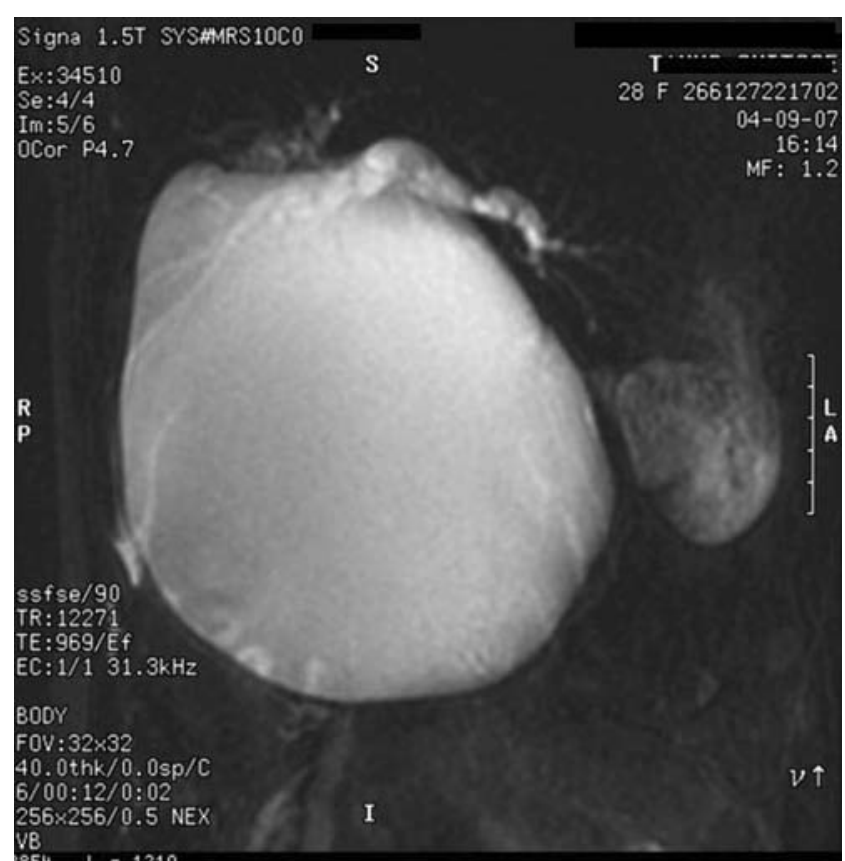

Fig. 3 MRCP of congenital dilatation of the common bile duct of a cystic form

However, there is some doubt that pancreaticobiliary maljunction causes dilatation of the bile duct based on the fact that some cases of pancreaticobiliary maljunction do not show dilatation of the bile duct and the experimental infusion of pancreatic juice into the bile duct of animals does not cause dilatation of the bile duct. Recently, congenital dilatation of the bile duct and pancreaticobiliary maljunction have been thought to be closely related to each other but to be different malformations.

Biliary tract carcinoma, especially bile duct carcinoma, is found in about $30 \%$ of patients with congenital dilatation

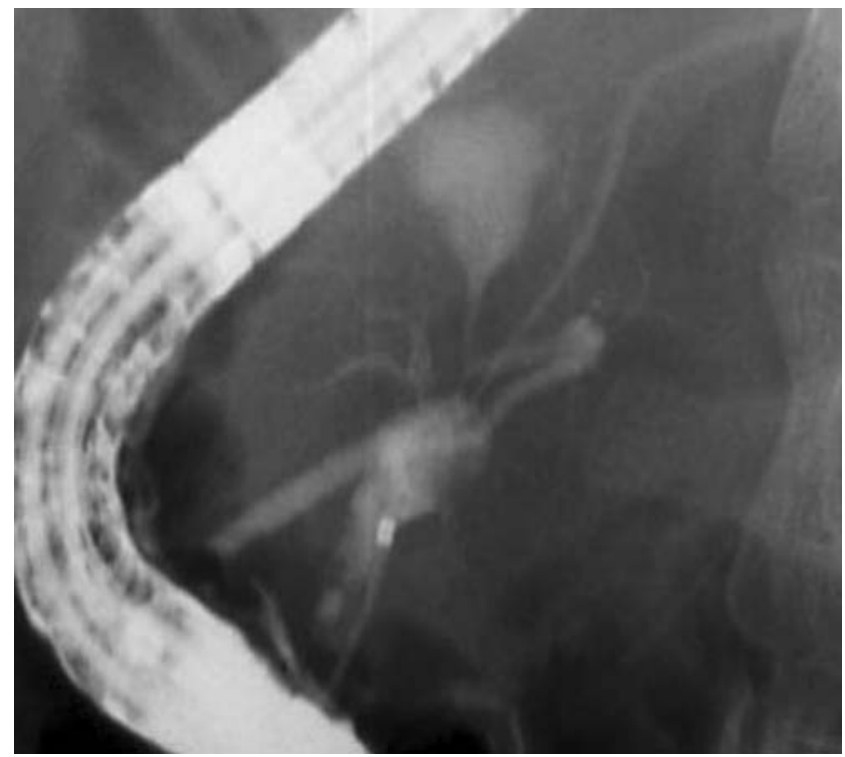

Fig. 4 ERCP findings of pancreaticobiliary maljunction 
of the bile duct. Pancreaticobiliary maljunction is thought to be related to its pathogenesis [6]. Thus, the concomitance of bile and pancreatic juice due to the inflow of pancreatic juice into the bile duct may produce powerful chemical substances. The stasis of such substances in the biliary tract is well known to induce cellular proliferation and reproduction and to stimulate genetic alterations in biliary epithelium, which may play an important role in carcinogenesis of the bile duct. In cases of pancreaticobiliary maljunction without dilatation of the bile duct, it has become clear that carcinoma can easily occur in the gallbladder which contains bile.

\section{Diagnosis}

In these cases, abdominal pain, jaundice, and abdominal mass are thought to be a triad. Abdominal pain is found in about $80 \%$ of cases, jaundice in $30-50 \%$, and abdominal mass in about $10 \%$. High fever, nausea, and vomiting accompanied by cholangitis or pancreatitis are also frequent. The important examinations for diagnosis are shown below.

\section{Examinations of the blood count and chemistry}

Hepatobiliary or pancreatic enzymes, such as pancreatic amylase, may be elevated. The white blood count or Creactive protein may be elevated due to cholangitis.

2. Abdominal ultrasound examination

Abdominal ultrasound is an important and easy examination for diagnosis. If this ailment is suspected, this examination should be performed soon.

3. Abdominal computed tomography (CT).

This procedure can provide very important information regarding not only the diagnosis but also the spread of dilatation and/or the coexistence of carcinoma and its spread and metastasis.

4. Endoscopic retrograde cholangiopancreatography

ERCP is useful for examining pancreaticobiliary maljunction (Fig. 4). It is also useful in the diagnosis of the extent of dilatation of the bile duct and its shape and for determining the spread of carcinoma.

5. Magnetic resonance imaging, MRCP (Figs. 1, 2, and 3)

The findings obtained by CT or ERCP can be acquired without any damage.

\section{Therapeutic strategies}

Due to the coexistence of carcinoma in about $30 \%$ of the cases, the basic strategy is surgery as soon as a diagnosis is made. The operation is dilated bile duct resection (Fig. 5) and hepaticojejunostomy, which ensure that pancreatic juice and bile do not mix in the bile duct. It is very important that pancreatic juice stays separated from bile to prevent the development of carcinoma of the bile duct.

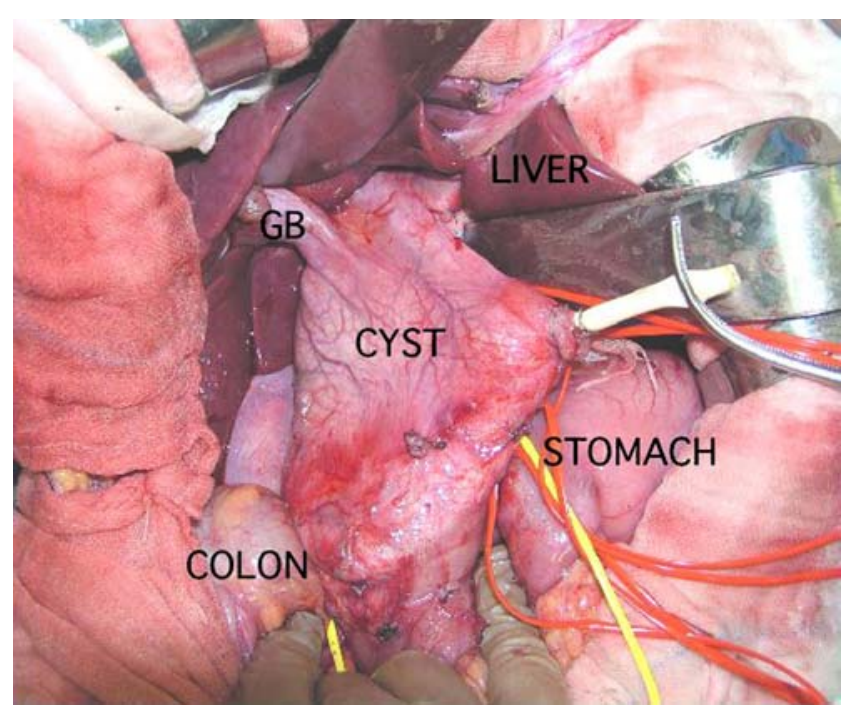

Fig. 5 Operative findings of congenital dilatation of the common bile duct of a cystic form

In cases with benign congenital dilatation of the common bile duct

Total removal of the dilated part of the bile duct is ideal but is not necessarily conducted in each case. With regard to the intrapancreatic bile duct, the bile duct is removed as much as possible. However, if the junction of the bile duct and pancreatic duct is too close, this may injure the main pancreatic duct and lead to severe postoperative complications such as acute pancreatitis or pancreatic fistula. The wall of the bile duct and the pancreatic parenchyma are strongly adhered to each other in most cases. Therefore, surgeons should not try to remove all of the dilated bile duct in the part covered by the pancreas. A 5- to $10-\mathrm{mm}$ wall of bile duct should be left in the intrapancreatic space from the junction of the bile duct and the pancreatic duct. The remaining wall of the bile duct in the pancreas should be sutured and closed.

Thus, on the duodenal side of the bile duct, only the mucosa of the bile duct which may develop carcinoma will be removed. Near the pancreaticobiliary junction, the mucosa of the bile duct is sutured by a purse-string suture. Near this suture, the fibrous thick wall of the bile duct is also sutured by a purse-string suture. Furthermore, the cut end of the bile duct is closed by interrupted sutures for complete closure of the duodenal side of the bile duct.

With regard to the hepatic side of the bile duct, the dilated part should be removed as much as possible. However, in cases with bilateral dilatation of both the right and left hepatic bile duct, complete resection of the dilated part of the bile duct is impossible. Furthermore, aggressive surgery should not be performed for benign lesions. Therefore, if there is no stenosis between intrahepatic bile ducts and either the right or left side of the hepatic duct, 
some dilated parts can be left and cut to prevent excess damage to the liver.

\section{In cases with dilatation of the bile duct with carcinoma}

An operation for carcinoma of the bile duct should be performed in cases with dilatation of the bile duct with carcinoma. The incision line of surgery should be set to remove all of the malignant parts. Lymph node dissection is also performed. Because the spread of carcinoma may not always be correctly diagnosed by imaging before the operation, an intraoperative histologic examination should also be used.

If carcinoma exists on the duodenal side of the bile duct, pancreaticoduodenectomy should be done. If carcinoma exists on the hepatic side of the bile duct, extended right or left lobectomy with resection of the caudate lobe should be performed. Portal embolization before the operation to enlarge the left hepatic lobe is sometimes necessary for a safe operation in extended right hepatic lobe resection.

\section{In cases with a past history of cystduodenostomy or cystjejunostomy}

In cases with a past history of cystduodenostomy or cystjejunostomy, a high incidence of carcinoma of the dilated bile duct may be seen by observation without operation. The dilated bile duct should be removed and hepaticojejunostomy should be performed as soon as possible to separate pancreatic juice from bile to prevent the development of carcinoma.

\section{Points of informed consent}

Patients should be persuaded that the development of cancer is possible in this ailment, and therefore surgery is absolutely necessary, even if they have no symptoms.

Patients should understand that retrograde cholangitis or intrahepatic duct stone formation may be possible and therefore long-term observation should be necessary, although the postoperative course is uneventful in most cases.

\section{Pancreaticobiliary maljunction}

Pancreaticobiliary maljunction is an anomalous condition in which the pancreatic duct and bile duct merge outside the duodenal wall. Because the sphincter Oddi does not function at the pancreaticobiliary junction, continuous pancreaticobiliary reflux may easily occur, which causes pancreaticobiliary diseases, such as cholangitis, stone formation, and/or gallbladder carcinoma [5] in the biliary tract and acute and/or chronic pancreatitis or pancreatic lithiasis. The development of carcinoma of the bile duct is a serious risk.

Congenital dilatation of the common bile duct has been shown to be very closely related to pancreaticobiliary maljunction. On the other hand, the bile duct is not dilated in some cases of pancreaticobiliary maljunction.

Congenital dilatation of the bile duct is described above. We now address pancreaticobiliary maljunction without bile duct dilatation.

Carcinoma of the bile duct is reported to occur in $30 \%$ to $40 \%$ of cases of pancreaticobiliary maljunction without dilatation of the bile duct. Gallbladder carcinoma develops in more than $90 \%$ of such instances.

Clinical symptoms Symptoms associated with complicating diseases, such as cholangitis, gallbladder stones, carcinoma in the biliary tract, acute or chronic pancreatitis and pancreaticolithiasis are seen.

Diagnosis The fact that the pancreaticobiliary junction is outside of the duodenal wall and therefore there is a very long common channel, and the fact that the form of the junction is abnormal, should be proven by ERCP or MRCP. High amylase values of bile in the bile duct or gallbladder are clues for diagnosis.

Early diagnosis is difficult in most cases of pancreaticobiliary maljunction without dilatation of the bile duct. However, pancreaticobiliary maljunction should be suspected and closely examined if thickness of the wall,

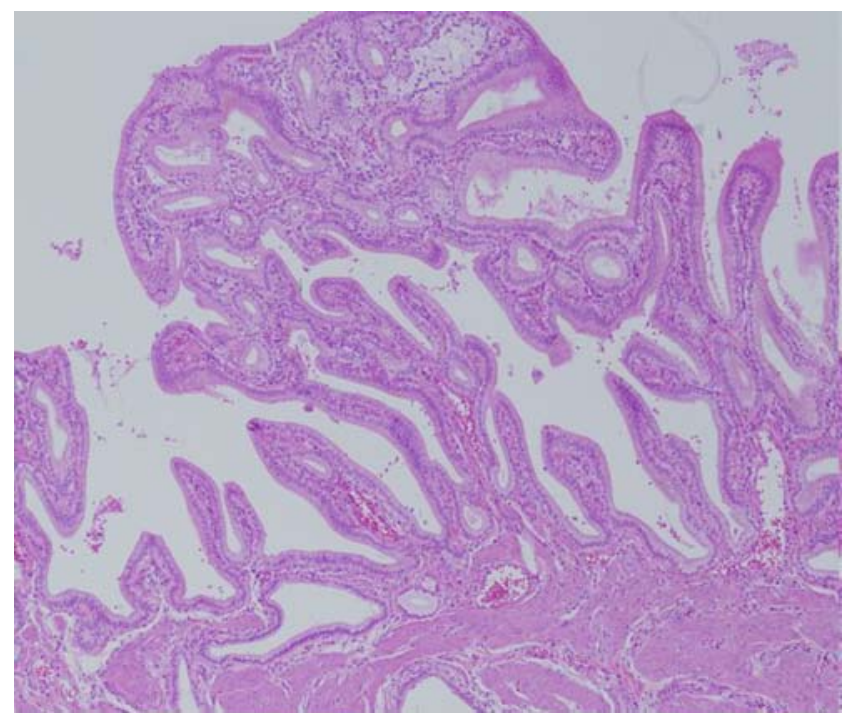

Fig. 6 Histological findings of papillary projection of epithelia of the gallbladder found in the cases with pancreaticobiliary maljunction 
especially epithelial thickness of the gallbladder (Fig. 6), is found by abdominal ultrasound, although no gallbladder stones were detected.

Therapeutic strategies Laparoscopic cholecystectomy should be performed due to the high frequency of concomitant gallbladder carcinoma. Surgery to separate the pancreatic juice and bile by bile duct resection and hepaticojejunostomy is controversial. The development of carcinoma in the bile duct in cases of pancreaticobiliary maljunction without dilatation of the bile duct is rare, and the high incidence of cholangitis after hepaticojejunostomy makes most doctors think that surgery for the separation of pancreatic juice and bile is not necessary [7].

Open Access This article is distributed under the terms of the Creative Commons Attribution Noncommercial License which permits any noncommercial use, distribution, and reproduction in any medium, provided the original author(s) and source are credited.

\section{References}

1. Kimura W (2005) Congenital dilatation of the common bile duct. Recent Ther Strateg 399-401, (in Japanese)

2. Alonso Lej F, Rever WB Jr, Pessagno DJ (1959) Congenital choledochal cyst, report of a 2 and an analysis of 94 cases. Int Abst Surg 108:1-29

3. Todani T (1997) Congenital choledochal dilatation. Classification, clinical features, and long term results. J Hepatobiliary Pancreat Surg 4:276-282

4. Babbit DP (1968) Congenital choledochal cysts: new etiological concept based on anomalous relationships of the common bile duct and pancreatic bulb. Ann Radiol 12:231-240

5. Anderson MC, Hagstrom WJ Jr (1962) A comparison of pancreatic and biliary pressures recorded simultaneously in man. Can J Surg 5:461-470

6. Kimura K, Ohto M, Saisho H, Unozawa T, Morita M, Ebara M, Matsutani S, Okuda K (1985) Association of gallbladder carcinoma and anomalous pancreaticobiliary duct union. Gastroenterology $89: 1258-1265$

7. Kobayashi S, Asano T, Yamasaki M, Kenmochi T, Saigo K, Ochiai $\mathrm{T}$ (2004) Prophylactic excision of the gallbladder and bile duct for patients with pancreaticobiliary maljunction. Arch Surg 136:759763 